Revue d'archéologie préhistorique

\title{
Les industries lithiques du Gravettien du nord de l'Aquitaine dans leur cadre chronologique
}

Gravettian lithic industries in Northern Aquitaine in their chronological

framwork

Jean-Philippe Rigaud

\section{(2) OpenEdition}

Journals

Édition électronique

URL : http://journals.openedition.org/paleo/1623

DOI : $10.4000 /$ paleo.1623

ISSN : 2101-0420

Éditeur

SAMRA

\section{Édition imprimée}

Date de publication : 1 décembre 2008

Pagination : 381-398

ISSN : $1145-3370$

\section{Référence électronique}

Jean-Philippe Rigaud, « Les industries lithiques du Gravettien du nord de l'Aquitaine dans leur cadre chronologique », PALEO [En ligne], 20 | 2008, mis en ligne le 13 mai 2011, consulté le 07 juillet 2020. URL : http://journals.openedition.org/paleo/1623 ; DOI : https://doi.org/10.4000/paleo.1623

\section{(c) $($ ) $(9)$}

PALEO est mis à disposition selon les termes de la licence Creative Commons Attribution - Pas d'Utilisation Commerciale - Pas de Modification 4.0 International. 


\title{
LES INDUSTRIES LITHIQUES DU GRAVETTIEN DU NORD DE L'AQUITAINE DANS LEUR CADRE CHRONOLOGIQUE
}

\author{
Jean-Philippe RIGAUD ${ }^{(1)}$
}

À Guy Célérier

\begin{abstract}
Résumé : Cet article se propose de montrer comment furent élaborés les modèles successifs du peuplement gravettien du sud-ouest de la France. De nombreux sites-clés, des archéoséquences exceptionnellement longues et détaillées et des recherches intensives conduites depuis le début du XX'̀ siècle, justifient le rôle primordial de l'Aquitaine dans la compréhension du peuplement gravettien de la façade atlantique de l'Europe. Le nord de l'Aquitaine est la région éponyme du Gravettien, Périgordien, Bayacien, Fontirobertien, Noaillien, Rayssien, Laugerien, Protomagdalénien, autant de faciès dont la signification culturelle, fonctionnelle ou chronologique est discutée. Une synthèse révisée est proposée dans le contexte européen du Gravettien.
\end{abstract}

Mots-clés : Aquitaine, Périgord, Gravettien, Périgordien, Bayacien, Fontirobertien, Noaillien, Rayssien, Laugerien, Protomagdalénien, chronologie.

Abstract: Gravettian lithic industries in Northern Aquitaine in their chronological framwork. This article is an attempt to describe how have been elaborated models for the peopling of south-western France. Large number of key-sites, exceptionally long and detailed archeosequences, intensives researches since the beginning of the $X X^{\text {th }}$ century, justify the crucial role of Aquitaine for understanding the Gravettian peopling of western Europe. Northern Aquitaine is the eponymous region for Gravettian, Périgordian, Bayacian, Fontirobertian, Noaillian, Rayssian, Laugerian, Protomagdalénian, all facies which cultural, functional or chronological meaning is criticized. An up dated synthesis is proposed within the European Gravettian context.

Key-words: Aquitaine, Perigord, Gravettian, Perigordian, Bayacian, Fontirobertian, Noaillian, Rayssian, Laugerian, Protomagdalenian, chronology.

\section{INTRODUCTION}

En dépit de leur forte variabilité, les industries gravettiennes partagent quelques caractères technologiques et typologiques qui justifient leur appellation générique de Gravettien. Leur dénominateur commun typologique est naturellement la pointe de La Gravette accompagnée souvent de sa forme réduite : la micropointe de La Gravette ou microgravette. Corrélativement, la fabrication des pointes de La Gravette implique la production de supports laminaires rectilignes et plats selon une méthode précise (Bordes 1968 ; Bordes et Crabtree 1969). En fonction de la qualité et de la morphologie des blocs de matières premières accessibles, les produits et sous-produits de cette chaîne opératoire sont variables, mais la présence de pointes ou micropointes de
La Gravette constitue le plus souvent une condition suffisante pour attribuer un assemblage à la culture gravettienne. Par contre, la technique du « dos abattu » ne constitue pas à elle seule un élément diagnostique du Gravettien tant qu'elle n'est pas mise en œuvre pour produire des pointes ou micropointes de La Gravette car cette technique fut très largement utilisée dans le monde entier, que ce soit dans le Middle Stone Age sud africain, le Paléolithique moyen européen, le Paléo-indien nord américain ou l'Epipaléolithique du Maghreb sans que l'on puisse évoquer une improbable influence gravettienne. À ce dénominateur commun que sont les pointes de La Gravette, présentes en quantités variables de l'Atlantique au Don s'ajoutent, selon le lieu ou le moment, des objets caractéristiques qui ont défini autant de faciès : fléchettes, burins de Noailles, du Raysse, pointes de

(1) Université Bordeaux 1 - Institut de Préhistoire et de Géologie du Quaternaire/PACEA, Avenue des Facultés, 33405 Talence - j.ph.rigaud@wanadoo.fr 


\begin{tabular}{|c|c|c|c|c|}
\hline & & SOLUTRÉEN & & \\
\hline AURIGNACIEN & & $\begin{array}{c}\text { PÉRIGORDIEN } \\
\text { SUPÉRIEUR } \\
\text { V a, b, c } \\
\text { La FERRASSIE }\end{array}$ & $\begin{array}{l}\text { PÉRIGORDIEN V } \\
\text { Type FONT ROBERT }\end{array}$ & $\begin{array}{l}\text { PÉRIGORDIEN V } \\
\text { Type NOAILLES }\end{array}$ \\
\hline $\begin{array}{l}\text { AURIGNACIEN } \\
\text { SUPÉRIEUR } \\
\text { Type La Gravette }\end{array}$ & $\begin{array}{c}\text { AURIGNACIEN } \\
\text { TYPIQUE ÉVOLUÉ V } \\
\text { LAUGERIE HAUTE }\end{array}$ & $\begin{array}{c}\text { PÉRIGORDIEN } \\
\text { SUPÉRIEUR IV } \\
\text { La Gravette }\end{array}$ & $\begin{array}{c}\text { PÉRIGORDIEN IV } \\
\text { Type La Gravette }\end{array}$ & $\begin{array}{l}\text { PÉRIGORDIEN à } \\
\text { Pointes de Font-Yves }\end{array}$ \\
\hline $\begin{array}{c}\text { AURIGNACIEN } \\
\text { MOYEN } \\
\text { Type CRO-MAGNON }\end{array}$ & $\begin{array}{c}\text { AURIGNACIEN } \\
\text { TYPIQUE I à IV } \\
\text { LA FERRASSIE }\end{array}$ & $\begin{array}{l}\text { PÉRIGORDIEN } \\
\text { MOYEN III } \\
\text { LAUGERIE-HAUTE }\end{array}$ & $\begin{array}{c}\text { PÉRIGORDIEN III } \\
\text { Type LAUGERIE- } \\
\text { HAUTE }\end{array}$ & $\begin{array}{c}\text { PÉRIGORDIEN à } \\
\text { Fléchettes } \\
\text { La Gravette }\end{array}$ \\
\hline $\begin{array}{c}\text { AURIGNACIEN } \\
\text { INFÉRIEUR } \\
\text { Type CHÂTELPERRON }\end{array}$ & & $\begin{array}{l}\text { PÉRIGORDIEN } \\
\text { INFÉRIEUR I, II } \\
\text { La FERRASSIE }\end{array}$ & $\begin{array}{c}\text { PÉRIGORDIEN I } \\
\text { Type } \\
\text { CHATELEPERRON }\end{array}$ & $\begin{array}{l}\text { PÉRIGORDIEN II } \\
\text { Type DUFOUR }\end{array}$ \\
\hline AURIGNACIEN & $\begin{array}{l}\text { AURIGNACIEN } \\
\text { TYPIQUE }\end{array}$ & PÉRIGORDIEN & $\begin{array}{l}\text { PÉRIGORDIEN } \\
\text { 1 }^{\text {er }} \text { Groupe }\end{array}$ & $\begin{array}{c}\text { PÉRIGORDIEN } \\
2^{\mathrm{e}} \text { Groupe }\end{array}$ \\
\hline H. Breuil & $\begin{array}{l}\text { D. Peyrony } \\
1933-1936\end{array}$ & & $\begin{array}{c}\text { D. Peyrony } \\
1946\end{array}$ & \\
\hline
\end{tabular}

Tableau 1 - L'Aurignacien et le Périgordien en Aquitaine selon H. Breuil et D. Peyrony (d'après L. Pradel In Sonneville-Bordes 1960).

Table 1 - Aurignacian and Perigordian in Aquitaine after H. Breuil and D. Peyrony in D. de Sonneville-Bordes (after L. Pradel in : Sonneville-Bordes 1960).

La Font-Robert, éléments tronqués, pointe à cran de Kostienki, pointes à face plane ou pointes foliacées bifaces, etc. sont autant de marqueurs de ces faciès typologiques. Enfin, en dépit de l'absence de pointes de La Gravette, certaines industries ont été parfois considérées comme gravettiennes parce qu'elles contenaient un ou plusieurs des marqueurs du cortège gravettien. En raison de la variabilité que nous venons d'évoquer, la répartition chronologique ou spatiale des faciès gravettiens a donné lieu à un certain nombre de modèles interprétatifs et classificatoires dont l'un des premiers fut celui de D. Peyrony.

\section{Le modèle aquitain proposé par D. Peyrony (1933-1950)}

Deux idées maîtresses étaient à la base du modèle Peyrony : d'une part la contemporanéité de l'Aurignacien et du Périgordien et, d'autre part, l'évolution linéaire du Périgordien ancien vers le Périgordien supérieur.

L'hypothèse de la contemporanéité de l'Aurignacien et du Périgordien était la conséquence, de la place que $D$. Peyrony mais aussi plus tardivement $\mathrm{F}$. Bordes, attribuait à l'Aurignacien entre le Périgordien ancien et le Périgordien supérieur. De plus, les caractéristiques typologiques de l'Aurignacien le séparant clairement de la lignée périgor- dienne, il ne pouvait s'agir d'un stade évolutif de cette dernière. Ils étaient donc nécessairement contemporains. Denis Peyrony supposait que le Périgordien I (Périgordien inférieur) était à l'origine du Périgordien moyen en se fondant sur l'usage d'une technique commune, celle du dos abattu, utilisée pour la fabrication des pointes de Châtelperron et des pointes de La Gravette et sur la présence de pointes de Châtelperron dans le Bayacien (Sonneville-Bordes 1960 :180). Ce point de vue fut longtemps partagé par F. Bordes (1968), F. Harold (1989), H. Bricker (1978).

Longtemps après les travaux de D. Peyrony (1933), de nombreux essais de synthèse se sont succédé pour proposer un modèle d'organisation des industries gravettiennes du nord de l'Aquitaine. Nous n'entreprendrons pas ici, une fois de plus, de faire l'historique du classement taxinomique du Paléolithique supérieur initial dont de nombreuses variantes ont été maintes fois publiées, accompagnées d'abondantes sinon complètes bibliographies auxquelles nous renvoyons (Sonneville-Bordes 1960 ; Bordes 1968 ; Delporte 1991 ; Rigaud 1982 ; Movius 1977 ; Bricker 1995 ; Otte et Noiret 2003 ; Bosselin et Djindjian 1994 ; Djindjian et Bosselin 1994 ; Soriano 1998 ; O'Farell 1996 ; Klaric 2003 ; Goutas 2004). Nous nous bornerons ici à rappeler que revient à $\mathrm{D}$. Peyrony le mérite d'avoir proposé en 


\begin{tabular}{|c|c|c|c|}
\hline La Gravette & Pataud & La Ferrassie & Laugerie-Haut-eEst \\
\hline & Protomagdalénien & & Protomagdalénien \\
\hline & Périgordien VI & & Périgordien VI (ex-III) \\
\hline & Noaillien récent & & \\
\hline & Noaillien ancien & Périgordien V c & \\
\hline & & Périgordien V b & \\
\hline & & Périgordien V a & \\
\hline $\begin{array}{c}\text { Périgordien IV } \\
\text { Bayacien }\end{array}$ & $\begin{array}{c}\text { Périgordien IV } \\
\text { Bayacien }\end{array}$ & & \\
\hline Aurignacien & Aurignacien & Aurignacien & \\
\hline
\end{tabular}

Tableau 2 - Archéoséquence du Gravettien ( Périgordien) en Dordogne d'après D. et E. Peyrony 1938 ; D. de SonnevilleBordes 1960 et H. L. Movius 1954.

Table 2 - Cultural sequence of the Gravettian (= Perigordian) in Dordogne after D. and E. Peyrony 1938 ; D. de SonnevilleBordes 1960 and H.L. Movius 1954.

1933 un premier modèle de classification de l'Aurignacien et du Périgordien dont l'ordonnancement, en l'absence de datations numériques, était fondé sur les archéoséquences de quelques sites du nord de l'Aquitaine (La Gravette, La Ferrassie, Laugerie-Haute-Est) et sur la comparaison typologique des industries lithiques et osseuses qu'elles contenaient.

Le tableau 1, emprunté à $D$. de Sonneville-Bordes (1960 : 35 ) résume clairement les conceptions successives de $D$. Peyrony.

\section{Nouvelles recherches (1960-2005)}

Le modèle de $\mathrm{D}$. Peyrony fut critiqué, souvent avec raisons mais aussi parfois sans considération pour le contexte dans lequel il fut élaboré. On lui a reproché notamment «...d'avoir retardé la connaissance du Gravettien français » (Bazile 2005 : 67), d'être « depuis plus de 60 ans [...] à la base d'un raisonnement erroné " (Bosselin et Djindjian 1994 :11) ou encore d'avoir été " abusivement imposée à d'autres régions, plus ou moins lointaines, voire à l'ensemble du continent eurasiatique. " (Delporte 1991 : 243). Au-delà de ces avis partiaux ou trop sévères, il convient de noter que ce modèle fut élaboré à partir des archéoséquences longues et détaillées des sites de La Gravette, de la Ferrassie, de Laugerie-Haute. De telles séquences étaient alors inconnues hors Aquitaine et le modèle proposé par $\mathrm{D}$. Peyrony devint une référence largement adoptée, parfois même abusivement transposée loin de la région éponyme.

L'analyse typologique et statistique des industries du Paléolithique supérieur, conduite par D. de SonnevilleBordes (1955a, 1955b, 1955c, 1960) fut décisive dans l'évolution du modèle proposé par D. Peyrony en proposant notamment de rattacher le Périgordien II à l'Aurignacien ancien (Sonneville-Bordes 1955a : 200). Le Périgordien III, observé à la base de la séquence de
Laugerie-Haute mais qui fut trouvé plus tard au-dessus du Périgordien V à l'Abri Pataud, devint le Périgordien VI (Movius 1954 ; Sonneville-Bordes 1960) (voir également les abondantes bibliographies mentionnées plus haut).

Finalement, ne subsistaient du Périgordien de D. Peyrony que les industries postérieures à l'Aurignacien : les Périgordiens IV, V, VI et le Protomagdalénien organisés selon le schéma du tableau 2.

Les travaux de H. L. Movius à l'Abri Pataud, de F. Bordes à Corbiac, de F. Bordes et J. Labrot au Roc-de-Combe, de H. Delporte à l'abri du Facteur et à la Ferrassie, de G. Célérier aux Jambes, J. et G. Guichard dans les sites de plein-air du Bergeracois et de nos propres fouilles au Flageolet 1 ont largement contribué à l'évolution du modèle Peyrony. Ces fouilles ont apporté des données nouvelles dans les domaines de la stratigraphie, de la chronologie des industries et des paléoenvironnements ainsi qu'une meilleure connaissance technologique et typologique des outillages lithiques et osseux qui remirent en cause certains points du modèle initial et conduisirent à une meilleure compréhension de la structuration du Gravettien périgourdin.

\section{1 - LE GRAVETTIEN MOYEN : LES PREMIÈRES MANI- FESTATIONS GRAVETTIENNES EN AQUITAINE}

La continuité entre le Périgordien ancien et le Périgordien supérieur était à la base du modèle Peyrony. Critiquée très tôt par D. Garrod (1938), puis par H. Delporte (1955), cette idée n'en fut pas moins soutenue tardivement par F. Bordes (1968), F. Harrold (1989), H. Bricker (1978) et nous-même pour quelque temps (Rigaud 1976). Mais, à la faiblesse des arguments techno-typologiques soutenant le point de vue de Peyrony, vint s'ajouter la découverte à Saint-Césaire d'une industrie castelperronienne associée à des restes de néandertalien, pour conduire à son abandon 
et au renoncement à rechercher une origine locale au Gravettien ${ }^{2}$. Le Périgordien moyen venait donc en discontinuité technologique et typologique prendre la suite de la séquence aurignacienne.

Les stratigraphies de La Gravette et de Pataud sont à la base de l'archéoséquence régionale du Gravettien. Rappelons qu'au sein du premier groupe Périgordien du modèle de D. Peyrony (cf. tableau 1), le Périgordien III succédait au Périgordien I type Châtelperron et, dans le second groupe, le Périgordien à fléchettes succédait au Périgordien II type Dufour (qui sera rattaché ensuite à l'Aurignacien). Le Périgordien à fléchettes du niveau moyen de l'abri de La Gravette, appelé Bayacien par F. Lacorre (1960) était donc la première manifestation gravettienne en Périgord ${ }^{3}$.

Les fouilles de H. Movius dans l'Abri Pataud, bien plus soigneuses que celles de F. Lacorre à La Gravette, ont mis au jour une archéoséquence particulièrement importante comportant de bas en haut (Bricker 1995) :

- niveau 5 : Périgordien moyen (Périgordien IV) avec à sa base le Bayacien ;

- niveau 4 : Noaillien (Périgordien supérieur ou Périgordien Vc à burins de Noailles) ;

- niveau 3 : Périgordien supérieur final (VI) ;

- niveau 2 : Protomagdalénien. ${ }^{4}$

\section{Le Périgordien moyen de l'Abri Pataud (Gravettien moyen)}

L'industrie du niveau 5 a été décrite par H. Bricker (1995) et nous renvoyons à cette publication pour plus de détails. Sur la base d'une analyse typologique et subtypologique (analyse d'attributs), $\mathrm{H}$. Bricker a distingué dans ce niveau, des variations mineures successives (Bricker 1995a). Ainsi, la base de ce niveau, surmontant directement la séquence aurignacienne, a livré des pointes de La Gravette et des fléchettes, ces dernières disparaissant lorsque l'on s'élève dans ce niveau. Cette particularité typologique, déjà remarquée à La Gravette avait incité $F$. Lacorre à évoquer une tradition bayacienne distincte de celle du Périgordien mais les données de Pataud montrent également qu'elles participent d'une même tradition technique et qu'il existe entre le Bayacien et le Gravettien moyen une continuité techno-typologique rattachant le faciès bayacien au Périgordien moyen dont il constitue la phase ancienne (Bricker 1995a : 165).

Les dates ${ }^{14} \mathrm{C}$ du niveau 5 de l'Abri Pataud (Bricker 1995), qui sont parmi les rares dont nous disposions actuellement pour le Périgordien moyen, sont comprises entre $28400 \pm 1100$ (Oxa 169) et $21780 \pm 215$ (GrN-4631) soit une durée approximative de 7000 ans. Nous devons noter cependant que les dates provenant de la partie avant de l'abri (GrN-4631, Ly 300, Ly 100, GrN5009, W 151 et W 191) sont nettement plus récentes que les autres dates de ce niveau ainsi que certaines du niveau 4 sus-jacent. Par contre, les dates provenant de la partie arrière et celles qui ont été obtenues pour les lentilles $\mathrm{K}-1, \mathrm{H}-3$, et $\mathrm{V}-2$ et $\mathrm{W}-1$, semblent indiquer un âge compris entre $28400 \pm 1100$ (Oxa 169) et $26600 \pm 200$ (Gr4477) plus conforme aux dates obtenues pour le reste de la séquence. Une révision stratigraphique détaillée du remplissage de cet abri pourrait révéler des perturbations post-dépositionnelles responsables d'une pollution des niveaux supérieurs de l'avant qui expliquerait un tel écart entre les dates.

Compte tenu de l'ancienneté des fouilles, l'association parfois évoquée de fléchette avec des pointes de La Font Robert, voire même avec des burins de Noailles, à Laussel, Durand-Ruel ou La Font-Robert est très probablement à mettre au compte d'une méthode de fouille archaïque et imprécise (voir aussi D. Pesesse, ce volume).

\section{3 - LE GRAVETTIEN SUPÉRIEUR EN AQUITAINE}

La séquence gravettienne se poursuit à Pataud par le niveau 4 attribué au "Noaillien » (Périgordien supérieur à burins de Noailles). Cependant, à la Ferrassie les fouilles de D. Peyrony avaient montré que, sous le Périgordien V à burins de Noailles (Vc), se trouvent d'autres niveaux attribués également au Périgordien $V$ par Peyrony : le Périgordien Va (à pointes de La Font-Robert) et le Périgordien $\mathrm{Vb}$ (à éléments tronqués) qui ne sont pas présents à Pataud (Peyrony 1934 ; Sonneville-Bordes 1960).

(2) Cette association était alors contradictoire avec l'hypothèse d'une continuité techno-typologique liant le Périgordien ancien et le Périgordien moyen et supérieur car, dans le modèle Peyrony, une continuité culturelle impliquait également une continuité anthropologique.

(3) Pour cette raison, il eut été logique de l'appeler Gravettien mais les profondes divergences opposant F. Lacorre et D. Peyrony jointes aux exigences du modèle linéaire de ce dernier (le Périgordien ancien n'existant pas à La Gravette) ont fait que le terme ne fut retenu ni par $D$. Peyrony, ni par ses successeurs (D. de Sonneville-Bordes et $F$. Bordes, $H$. Delporte, H. L. Movius, J. Sackett, H. Bricker, N. David, B. Clay, J.-Ph. Rigaud, etc.) Toutefois, il est évident que les termes de Périgordien et Gravettien ne représentent plus depuis longtemps des entités culturelles différentes contrairement aux affirmations de Djindjian F. et Bosselin B., (1994: 117) tout au plus peut-on voir dans le maintien de leur usage les effets d'une «habitude» des préhistoriens oeuvrant en Périgord où il est notoire que le Périgordien est le faciès périgourdin du Gravettien européen.

(4) Contrairement à Djindjian et Bosselin (1994) et par respect pour l'opinion de nos collègues, nous avons conservé ici la dénomination et les attributions culturelles des industries qu'ils ont mises au jour, étudiées et publiées. En modifiant arbitrairement comme l'ont fait Djindjian et Bosselin, la dénomination des ensembles lithiques il est évidemment plus facile de donner l'impression d'une apparente logique et de satisfaire ainsi aux objectifs d'une " restructuration » qui demeure avant tout essentiellement fondée sur une documentation livresque, sélective et dépourvue de toute évaluation taphonomique. 


\begin{tabular}{|c|c|c|c|}
\hline Pataud & Roc de Combe-Capelle & La Ferrassie & La Gravette \\
\hline $\begin{array}{l}\text { Périgordien } \\
\text { supérieur à burins } \\
\text { de Noailles }\end{array}$ & & $\begin{array}{l}\text { Périgordien V à burins } \\
\text { de Noailles }\end{array}$ & \\
\hline & & $\begin{array}{l}\text { Périgordien V à } \\
\text { éléments tronqués }\end{array}$ & \\
\hline & $\begin{array}{l}\text { Périgordien V à } \\
\text { pointes de la Font } \\
\text { Robert (couche G) }\end{array}$ & \multirow[t]{2}{*}{$\begin{array}{l}\text { Périgordien V à pointes } \\
\text { de la Font Robert }\end{array}$} & \\
\hline $\begin{array}{l}\text { Périgordien IV } \\
\text { Bayacien }\end{array}$ & $\begin{array}{c}\text { Périgordien IV à } \\
\text { pointes de La Gravette } \\
\text { (couche E) }\end{array}$ & & $\begin{array}{l}\text { Gravettien } \\
\text { Bayacien }\end{array}$ \\
\hline Aurignacien & $\begin{array}{c}\text { Aurignacien } \\
\text { (couche C) }\end{array}$ & Aurignacien & Aurignacien \\
\hline
\end{tabular}

Tableau 3- Archéoséquences du Gravettien moyen et supérieur dans le nord de l'Aquitaine.

Table 3 - Cultural sequence of the Middle and Upper Gravettian in northern Aquitaine.

La reprise des fouilles (1968-1973) à La Ferrassie par H. Delporte a montré une stratigraphie dans laquelle, succédant à l'Aurignacien, plusieurs niveaux Périgordiens qui correspondaient globalement à ceux qui avaient été observés par D. Peyrony :

- D2 Sud (D2a, D2c, D2e, D2g), D2 Nord (E1 frontale, D2 sagittale, Dé-D3 frontale) contenant le Périgordien à pointes de La Font Robert ;

- D1-C4 contenant le Périgordien à éléments tronqués ;

- B4-B3 avec quelques traces de Périgordien à burins de Noailles.

Au Roc-de-Combe-Capelle ${ }^{5}$, à la suite des fouilles anciennes (1907) peu méthodiques de deux amateurs locaux J. Esclafer et M. Villeréal, D. Peyrony avait relevé une stratigraphique et étudié les industries qui avaient été recueillies niveau par niveau. L'archéoséquence suivante fut décrite de bas en haut (Peyrony 1943 :158-173) :

- A un niveau de «Périgordien I » (Castelperronien)

- B niveau stérile

- C un niveau Aurignacien

- D niveau stérile
- E Périgordien IV à pointes de La Gravette

- $F$ niveau très pauvre

- G niveau noir épais de Périgordien $V$ à pointes de la Font Robert.

L'industrie de la couche $E$ était comparable selon $D$. Peyrony à celle de l'abri de La Gravette. Bien que D. Peyrony ait formulé quelques réserves au sujet de la méthode de fouilles, la succession du Périgordien IV et Périgordien $\mathrm{V}$ observée par les fouilleurs, ne semblait pas lui poser de problème.

Le tableau 3 résume les conclusions auxquelles conduisent la comparaison de ces séquences et montre que le Périgordien IV à pointes de La Gravette se trouve sous le Périgordien Va à pointes de La Font-Robert au Roc-deCombe-Capelle et que les Périgordiens $\mathrm{Vb}$ et $\mathrm{Vc}$ de $\mathrm{D}$. Peyrony lui succèdent à La Ferrassie et pro parte à Pataud.

Les rares datations ${ }^{14} \mathrm{C}$ dont nous disposons pour cette période semblent confirmer cette succession :

- le Périgordien moyen (Périgordien IV à fléchettes) à la base du niveau 5 de l'Abri Pataud, compte tenu des

(5) Le Roc-de-Combe-Capelle est un abri-sous-roche à proximité de l'abri du Haut de Combe-Capelle. Ces deux abris dominent Combe-Capelle Bas, un important dépôt de pente fouillé successivement par D. Peyrony, H.-M. Ami, H. Dibble et M. Lenoir (H. L. Dibble and M. Lenoir 1995). 
remarques formulées précédemment, pourrait se voir attribuer un âge compris entre $28150 \pm 225$ BP (Gr 4634) et 28 $400 \pm 1100$ BP (Oxa 169).

- le Périgordien Va (à pointes de La Font Robert) de la Ferrassie (D2, E1) $27900 \pm 770$ BP (Oxa 402), $27530 \pm 720$ BP (Oxa 403) et $26250 \pm 620$ BP (Oxa 404) auxquelles il convient d'ajouter les dates ignorées par Bosselin et Djindjian (1994) : $23960 \pm 550$ BP (Gif 2696), $24650 \pm 550$ BP (Gif 2698) et $22520 \pm 500$ BP (Gif 2699) (Delibrias in Delporte, 1984).

De plus, les dates de $29350 \pm 360$ BP (Beta 145820) et 27300 \pm 330 BP (Oxa 10820) obtenues récemment par F. Surmely dans le site de Sire (Allier) pour une industrie de Périgordien à pointes de La Gravette (Surmely, ce volume) viennent soutenir, s'il en était besoin, l'existence d'un Périgordien à pointes de La Gravette antérieure à celle d'un Périgordien à pointes de La Font-Robert et contredit ainsi les hypothèse formulées par Djindjian et Bosselin (1994) et Otte et Noiret (2003).

En outre, il convient de rappeler que les niveaux contenant des pointes de La Font-Robert au Flageolet I, rebaptisés (restructuration oblige !) « Gravettien indifférencié 》 par Djindjian et Bosselin (1994:119) ont donné un âge postérieur à $27000 \mathrm{BP}$ : Flageolet 1 , VII : $26150 \pm 600$ et Flageolet 1 , VI : $26500 \pm 900$ (Oxa 579) et $24280 \pm 500$ (Ly 2722)

\section{4 - LES FACIÈS DU GRAVETTIEN SUPÉRIEUR (EX-PÉRIGORDIEN V)}

Les stratigraphies de La Ferrassie, de l'Abri Pataud, du Flageolet 1 et de la Grotte XVI sont à la base de la séquence du Gravettien supérieur du nord de l'Aquitaine.

\section{Les données de La Ferrassie}

Au-dessus des derniers niveaux aurignaciens, D. Peyrony avait observé un ensemble gravettien dans lequel, à la faveur d'une stratigraphie plus claire dans la partie Est de l'abri, il avait par la suite distingué trois subdivisions : les niveaux $\mathrm{J}$, $\mathrm{K}$ et $\mathrm{L}$ respectivement caractérisés par trois fossiles directeurs : les pointes de La Font-Robert (J), les éléments tronqués $(K)$ et les burins de Noailles $(L)$. Ces subdivisions n'avaient pas été observées au début des fouilles, mais à l'issue de ses fouilles D. Peyrony, sur la base d'observations tardives, avait « redistribué » les fossiles directeurs présents dans la série globale initiale, dans les assemblages provenant des niveaux $\mathrm{J}, \mathrm{K}$, et $\mathrm{L}$ (Sonneville-Bordes 1960 :193). Cet enrichissement artificiel avait incité D. de Sonneville-Bordes à exprimer quelques réserves sur la représentativité typologique des séries provenant de ces niveaux, allant jusqu'à ne pas dénombrer les éléments tronqués dans l'inventaire typologique de la couche $\mathrm{K}$ (Sonneville-Bordes, loc.cit.).

Les fouilles plus récentes de $\mathrm{H}$. Delporte ont permis de recueillir dans l'équivalent de la couche $\mathrm{J}$ de Peyrony, une industrie plus représentative permettant de définir l'équilibre typologique du Périgordien à pointes de La FontRobert (Delporte 1984). Ce dernier est caractérisé par l'abondance des grattoirs qui, exceptionnellement dans le Gravettien, sont ici plus nombreux que les burins (Sonneville-Bordes loc. cit., Delporte loc.cit.). Les pointes de La Gravette et microgravettes sont bien représentées. L'originalité de cette industrie a incité $\mathrm{H}$. Delporte à l'individualiser sous le terme de « Fontirobertien » dans lequel il distinguait :

- le faciès présent à La Ferrassie (Fontirobertien stricto sensu) dont les pointes de La Font-Robert étaient du « type ancien » (Delporte et Tufffreau 1976, Bosselin et Djindjian 1994) ;

- le faciès du type Roc-de-Combe et Flageolet I (niveaux VII et $\mathrm{VI}$ ) dans lequel les pointes de La Font-Robert sont moins abondantes et d'un type " plus évolué » et associées à quelques burins de Noailles (Delporte et Tuffreau loc.cit. ; Bosselin et Djindjian loc. cit .). Selon H. Delporte, le second faciès pourrait être légèrement plus récent que le Fontirobertien stricto sensu mais les corrélations climatiques sur lesquelles il se fondait pour établir cette antériorité ne sont plus acceptable actuellement (Delporte 1984 : 245).

Les dates ${ }^{14} \mathrm{C}$ obtenues pour cette industrie de La Ferrassie sont les suivantes :

Coupe sagittale D2:24 $650 \pm 550$ (Gif 2698)

D2 : $23960 \pm 550$ (Gif 2696)

D2 : $22520 \pm 500$ (Gif 2699)

Coupe frontale D2 : $27900 \pm 770$ (Oxa 402)

D2 : $27530 \pm 720($ Oxa 403)

$\mathrm{E} 1: 26250 \pm 620($ Oxa 404)

II y a de toute évidence une forte discordance entre les dates obtenues à partir des échantillons issus de la coupe sagittale et ceux de la coupe frontale, ces derniers donnant des âges beaucoup plus anciens que les premiers. Nous avons développé ailleurs (Delpech et Rigaud 2001) les conséquences de cette remarque qui sont de plus en cohérence avec les résultats de l'analyse du processus de formation du site réalisée par J.-P. Texier (2001) qui concluait que ces différentes anomalies sont probablement liées en grande partie à des problèmes de dynamique sédimentaire et qu'« il paraît hasardeux de se fonder sur ces archéostrates pour définir des stades d'évolution culturels »(Texier loc. cit. p. 172) ${ }^{6}$.

(6) De plus, les fouilles de H. Delporte à La Ferrassie se sont limitées aux coupes sagittales et frontales laissées par D. Peyrony, soit deux bandes étroites dont la surface totale est inférieure au $1 / 200^{\mathrm{ème}}$ de la surface évaluée du site. Nous en avions conclu (Rigaud 1982, 1988) que lors des fouilles H. Delporte, conçues alors comme un « rafraîchisssement de coupe (Delporte 1995, p. 20), les assemblages recueillis n'étaient pas archéologiquement (et non statistiquement comme le soutint Djindjian) représentatifs du contenu initial eu égard aux fréquentes variations quantitatives et qualitatives que l'on observe dans la répartition horizontale des vestiges. Ce manque de représentativité avait d'ailleurs été reconnu par $H$. Delporte (1984 : $273 ; 1994$ : 247-248). 


\section{Les données de l'Abri Pataud}

Le niveau 4 de l'Abri Pataud contient une industrie abondante qui fut l'objet d'une description détaillée de la part de $\mathrm{N}$. David (David In Bricker 1995) à laquelle nous renvoyons. Subdivisées en 3 ensembles successifs, cette industrie comporte moins de grattoirs que de burins et moins de burins dièdres que de burins sur troncature. Au sein de ces derniers, les burins de Noailles diminuent en nombre vers le sommet (de $37,6 \%$ à 2,4\%) alors que inversement les burins du Raysse augmentent (de 0,3\% à 30,3\%). Les pointes de La Gravette et les microgravettes sont peu nombreuses mais présentes. Les pièces tronquées ou bitronquées sont rares et les pointes de La Font-Robert absentes.

\section{Les données du Flageolet I}

Fouillé de 1966 à 1982 (Rigaud 1982b ; Lucas 2000), cet abri a livré une stratigraphie dans laquelle, surmontant un ensemble de niveaux aurignaciens, la séquence gravettienne du Flageolet I débute par:

- le niveau VII dans lequel sont présents quelques burins de Noailles (1,9\%), des pointes de La Gravette $(7,1 \%)$ et microgravettes $(3,8 \%)$, quelques éléments tronqués $(0,7 \%)$ et quelques pointes de La Font-Robert $(0,8 \%)$. Les grattoirs $(22,10 \%)$ sont plus nombreux que les burins $(14,6 \%)$ et l'ensemble des pièces à dos représente $15,2 \%$. Les dates ${ }^{14} \mathrm{C}$ obtenues pour ce niveau sont : >25 $720 \pm 610$ (Ly 1748) et $26150 \pm 600$ (Ly 2723) ;

- le niveau VI qui lui est superposé a livré quelques burins de Noailles (1,9\%), quelques burins du Raysse $(0,48 \%)$, des Gravettes $(1,29 \%)$ et des microgravettes (3\%), des éléments tronqués $(4,36 \%)$ et quelques pontes de La Font-Robert $(0,48 \%)$. Les grattoirs $(14,05 \%)$ sont très légèrement moins nombreux que les burins (18,90 \%) et l'ensemble des pièces à dos représente $13,89 \%$. Deux dates ${ }^{14} \mathrm{C}$ ont été obtenues : $24280 \pm 500$ (Ly 2722) et $26500 \pm 900$ (Oxa 447);

- le niveau $\mathrm{V}$ comporte des burins de Noailles (2,7 \%), des burins du Raysse (6,5 \%), quelques pointes de La Gravette $(0,2 \%)$ et microgravettes $(0,2 \%)$, des éléments tronqués, $0,59 \%)$. II n'y a pas de pointes de La Font-Robert. Les grattoirs $(8,1 \%)$ sont très nettement moins nombreux que les burins (54,5\%). Les pièces à dos ne représentent que 1,78 $\%$. Ce niveau a donné deux dates ${ }^{14} \mathrm{C}: 22520 \pm 500$ (Ly 2721) et $25700 \pm 700$ (0xa 447);

- le niveau IV présente un équilibre comparable au précédent. Les burins de Noailles sont rares $(0,57 \%)$, les burins du Raysse mieux représentés $(3,44 \%)$, les pointes de La Gravette (1,7\%) et microgravettes $(0,57 \%)$ peu abondantes. Les grattoirs (8,5\%) sont bien moins nombreux que les burins (53,5 \%) et les pièces à dos ne dépassant pas 3,4\%. Trois dates ${ }^{14} \mathrm{C}$ ont été obtenues pour ce niveau $>21190 \pm 920$ (Ly
1607), $22950 \pm 500$ (Ly 2186) et $23250 \pm 500$ (Oxa 596) ; - le niveau I-III, comporte des burins de Noailles $(0,57 \%)$, des burins du Raysse $(0,57 \%)$ des pointes de La Gravette $(1,14 \%)$ et des microgravettes (1,14\%). II n'a pas d'éléments tronqués et par de pointes de La Font-Robert. Les grattoirs (11,46\%) sont bien moins nombreux que les burins $(31,61 \%)$. Les pièces à dos représentent $5,17 \%)$. Ce niveau a donné 3 dates : $>22$ $440 \pm 680$ (Ly 1606), $24600 \pm 700$ (Oxa 448) et $18610 \pm 440$ (Ly 2185) mais cette dernière date, nettement plus récente que le reste de la séquence, pourrait indiquer une pollution.

\section{Les données de la Grotte XVI}

Réalisées en collaboration avec J. Simek, les fouilles dans cette grotte (1982-2001) ont mis au jour une séquence où, superposés à des occupations moustériennes et castelperronienne, se succédaient des niveaux aurignaciens, gravettiens, solutréen et magdalénien (Rigaud et Simek 1993).

Le niveau gravettien, pour lequel la date de $26840 \pm 470$ (AA2670) a été obtenue, contient une industrie où les grattoirs $(10,8 \%)$ sont légèrement plus nombreux que les burins $(6,18$ $\%$ ) et les burins dièdres plus nombreux que les burins sur troncature. Les pointes de La Gravette $(5,15 \%)$ et les microgravettes $(6,18 \%)$ sont présentes. Les éléments tronqués représentent $8,76 \%$ de l'outillage. II y a une pointe de La FontRobert mais les burins de Noailles sont totalement absents. Par sa composition typologique, cette industrie gravettienne se rapproche de celles de la couche K (fouilles Peyrony) et C4-D1 (fouilles H. Delporte) de la Ferrassie qui représentent le Périgordien $\mathrm{Vb}$ (à éléments tronqués) du modèle Peyrony.

Mal connue et rarement décrite cette industrie fut rejetée par B. Bosselin et F. Djindjian (1994) sous prétexte que: «... les industries rapportées au Périgordien IV et les industries rapportées au Périgordien Vb (à éléments tronqués) appartiennent systématiquement et obstinément au même faciès typologique! » (Bosselin et Djindjian 1994 : 119). Mais en poursuivant la lecture la cause devient évidente : "Cette fameuse couche à pointes de La Gravette et à " éléments tronqués 》 (qui sont des pointes de La Gravette tronquées à une ou deux extrémités) n'a été reconnue et caractérisée qu'à la Ferrassie, aux Vachons et à Laraux. Elle n'est pas réellement identifiable par la seule présence des éléments tronqués (dont l'effectif n'est sans doute lié qu'à la richesse en pointes de La Gravette)» (Djindjian et Bosselin 1994 : 119). II est certain qu 'en débaptisant les «éléments tronqués " pour en faire des pointes de La Gravette (tronquées ou bitronquées), le faciès à éléments tronqués ne résistera pas à une analyse statistique élémentaire, mais cette manipulation n'est possible qu'en ignorant les différences morphologiques et technologiques évidentes qui existent entre les pointes de La Gravette et les « éléments tronqués » et qui ont été décrites?.

(7) L'analyse comparative de la morphologie des éléments tronqués et des pointes de La Gravette montre clairement que les supports sont le plus souvent minces et de section trapézoïdale pour les premiers, épais et triangulaires pour les seconds, que la retouche du dos des premiers est semi-abrupte et peu importante alors que le dos des pointes de La Gravette est généralement abrupte et épais. Bosselin et Djindjian (1994 : 119) poursuivent en affirmant : "Par ailleurs il existe des éléments tronqués dans les niveaux traditionnellement attribués au Périgordien IV, (Rigaud 1983) » ce qui est une contre-vérité et nous n'avons jamais écrit cela puisqu'il n'y jamais eu de Périgordien IV au Flageolet. Il est probable que ces auteurs ont voulu évoquer l'industrie de la couche VII dans laquelle nous avons publié (Rigaud 1982b) la présence de pointes de La Font-Robert, d'éléments tronqués et de burins de Noailles. 
C'est la forte variabilité techno-typologique que l'on peut observer dans les industries du Périgordien supérieur qui est à l'origine de l'apparition d'entités taxinomiques destinées à souligner leurs particularités : le «Fontirobertien », le « Noaillien » et le « Rayssien ».

\section{Le Fontirobertien}

H. Delporte et A. Tuffreau (1976 : 245) ont créé le terme de Fontirobertien pour désigner les industries gravettiennes à pointes de La Font-Robert. Ces industries montrent effectivement des caractères qui leur sont propres comme le rapport grattoirs / burins, et la présence de pointes à pédoncule de type Font-Robert, mais elles comportent également en quantité notable les éléments marqueurs de la culture gravettienne à savoir les pointes et micropointes de La Gravette. Le Fontirobertien demeure un faciès typologique d'une industrie gravettienne.

Bosselin et Djindjian ont soutenu l'hypothèse de l'antériorité du faciès à pointes de La Font-Robert sur le Périgordien à pointes de La Gravette (Périgordien IV) en se fondant sur deux arguments. D'une part, les datations ${ }^{14} \mathrm{C}$ de la Ferrassie, mais nous avons vu plus haut qu'il convient d'être extrêmement prudent sur ce point (Delpech et Rigaud 2001 ; Texier 2001). D'autre part, sur une ancienneté du faciès hennuyer du Fontirobertien (Haeserts et Heinzelin 1979 ; Otte 1979) établie, il faut le souligner, sur des corrélations paléoclimatologies indirectes que Delporte et Tuffreau (1976 : 245) avaient prudemment pondérées et qui n'ont plus de valeur actuellement. De plus, ce faciès hennuyer n'a que de très lointaines ressemblances avec le Périgordien à pointes de La Font-Robert aquitain ; en fait leur seul point commun est la présence de pointes de La Font-Robert, mais d'autres caractères les séparent, notamment le rapport grattoirs / burins inverse de celui qui caractérise le Périgordien à Font Robert du sud-ouest de la France. $\mathrm{H}$. Delporte et A. Tuffreau (loc.cit.) avaient d'ailleurs formulé de sérieuses réserves en affirmant que l'industrie « $d u$ Périgordien hennuyer présente certaines caractéristiques qui la rapprochent plus, si l'on excepte les pointes de La Font-Robert, des gisements de l'Europe centrale que ceux du sud-ouest de la France " (Delporte et Tuffreau loc. cit. $p$ 245). Trop d'incertitudes chronologiques et des corrélations typologiques peu convaincantes ne permettent pas de retenir l'hypothèse de B. Bosselin et F. Djindjian.

\section{Le Noaillien}

Au vu des particularités typologiques et sub-typologiques (analyse d'attributs) de l'industrie lithique de la couche 4 de l'Abri Pataud N. David (1985) avait proposé de l'isoler du Périgordien pour en faire une « civilisation noaillienne indépendante » (David In Bricker loc. cit. p.130). Ce « Noaillien » comportant toutefois un certain nombre de traits typologiques et technologiques propres au Gravettien, comme la présence faible mais systématique de pointes de La Gravette et de microgravettes, nous avions contesté l'interprétation de N. David (Rigaud 1978, 1982) et proposé de considérer le « Noaillien » comme un faciès techno-typologique du Gravettien montrant effectivement quelques caractères propres mais également une variabilité indéniable, notamment dans l'importance relative des fossiles directeurs. Pour David (loc. cit.) la présence d'éléments gravettiens (pointes de La Gravette et microgravettes) est le résultat d'un processus d'acculturation d'une tradition noaillienne dans un monde gravettien. Mais, dans ce cas, il devrait y avoir un foyer noaillien non acculturé or à ce jour nous n'avons aucune indication de son existence. Le "Noaillien » représente donc un faciès typologique du Gravettien dont la composition montre une variabilité à laquelle nous avons proposé de donner une cause fonctionnelle (Laville et Rigaud 1973 ; Rigaud 1978). La recherche des causes de cette variabilité dans la fonction des sites par l'étude de la saisonnalité, des stratégies d'acquisition alimentaires et techniques et de l'organisation spatiale inter-site et intra-site nous semblent des voies de recherche plus prometteuses que l'évocation d'un processus d'acculturation préhistorique dont les critères d'évaluation (stylistique ? typologique ? technologique? symbolique ?) et les modalités n'ont jamais été décrites clairement (Rigaud 1978, 1985) ${ }^{8}$.

\section{Le Rayssien}

En fin de la séquence noaillienne de l'Abri Pataud, N. David (David in Bricker 1995) avait remarqué un remplacement progressif des burins de Noailles par les burins du Raysse. II en était de même dans plusieurs sites aquitains (Flageolet 1, Les Jambes, Solvieux, Fourneau du Diable, etc.) Pour cette raison, nous avions à maintes reprises souligné que, au Flageolet comme ailleurs, le remplacement des burins de Noailles par les burins du Raysse se faisait systématiquement en fin de séquence noaillienne et qu'il s'agissait de ce fait d'une émergence liée à la chronologie (Laville et Rigaud 1973 : 336 ; Rigaud 1976, 1978, $1982 b$, versus Klaric $2003: 22$ ).

En se fondant sur les données du site de la Picardie (Indre-etLoire, France), de Plasenn'Al-Lomm (Île de Bréhat, Côtes d'Armor) et des Artigaux (Gironde) où avaient été mises au jour des industries à nombreux burins du Raysse, totalement dépourvue de pointes et micropointes de La Gravette, L. Klaric (2003) a proposé une hypothèse selon laquelle il y aurait une tradition technique originale « rayssienne » distincte de la

(8) L'usage par les préhistoriens du concept d'acculturation, issu de l'anthropologie culturelle, s'est largement développé au cours des dernières décades sans avoir été réellement argumenté. Pour certains (David 1995 ; Mellars 1989) il explique les changements soudains de comportement des Gravettiens ou des Castelperroniens, pour d'autres ce concept « justifie » les incohérences d'un modèle hasardeux. Récemment un scénario surprenant fut proposé dans lequel «...des groupes humains porteur d'outillages à faciès aurignacien évolué [auraient] migré vers le sud sous la pression climatique du Pléniglaciaire supérieur, puis développé des cultures Gravettiennes, par acculturation avec d'autres populations locales aurignaciennes » (Djindjian et al. 1999 : 182 ; Péan 2001: 20). 
lignée périgordienne. Mais pour étayer cette hypothèse il fallait également montrer que, contrairement à ce qui était communément admis, toutes les industries à burins du Raysse, à l'instar de celle de la Picardie, étaient dépourvues des marqueurs gravettiens (pointes de La Gravette et microgravettes, Noailles, pointes de La Font-Robert, éléments tronqués) et qu'il n'y avait pas de continuité techno-typologique entre les industries à burins de Noailles et celles qui contiennent des burins du Raysse. L. Klaric entreprit alors une analyse taphonomique des archéoséquences de quelques sites du nord de la France afin de vérifier si la présence de pointes de La Gravette dans certains assemblages « rayssiens » ne pouvait pas être mise au compte de mélanges post-dépositionnels. Dans la Grotte du Renne à Arcy-sur-Cure, L. Klaric, (2003 : 168-185) a argumenté une possibilité de pollution des industries à burins du Raysse par un niveau Gravettien sus-jacent supposant ainsi que cette industrie serait de fait, comme celle de la Picardie, dépourvue de pointes et micropointes de La Gravette . Se fondant sur cette première hypothèse, L. Klaric suggère que de telles pollutions auraient de façon plus générale affecté l'homogénéité des industries à burins du Raysse contenant des pointes de La Gravette et des microgravettes et plus particulièrement celles qui proviennent de dépôts en grotte ou sous abris (Klaric $2003: 392)^{9}$ et sans remettre clairement en cause l'intégrité et l'homogénéité des archéoséquences du Flageolet et de l'Abri Pataud, L. Klaric (2003 : 392) estime nécessaire qu'elles fassent l'objet d'une évaluation taphonomique.

Conscients de l'importance d'une telle évaluation dans un abri-sous-roche à stratigraphie complexe, nous avions dès 1969 (Rigaud 1969, 1994) mis en œuvre des moyens de contrôle appropriés afin de tester l'homogénéité des nappes de vestiges mises au jour ${ }^{10}$ et nous avons été conduits à procéder à des corrections d'attribution stratigraphique, mais l'existence dans ces industries des marqueurs gravettiens et des burins du Raysse a été confirmée. La présence effective de pointes de La Gravette et de microgravettes dans les industries « rayssiennes » du Flageolet 1 va donc à l'encontre de la proposition de L. Klaric et confirme ce qui a été observé à l'Abri Pataud, aux Jambes, aux Fieux (Guillermin ce volume) et à Solvieux (Sackett 1999) ${ }^{11}$.

\section{5 - L'INTERPRÉTATION DES FACIÈS TYPOLOGIQUES DU GRAVETTIEN SUPÉRIEUR}

La révision chronologique du Gravettien français proposée par B. Bosselin et F. Djindjian (1994 ; Djindjian et Bosselin
1994) repose de fait sur des inventaires exclusivement bibliographiques qui n'ont pas été nuancés par une fréquentation plus intime du matériel archéologique (Rigaud 2000a, 2000b ; Klaric 2003 ; Goutas 2004). Tous les efforts classificatoires contenus dans leur démarche reposent sur une conception étroite de la signification de la variabilité typologique et quantitative des industries préhistoriques. La mise en évidence de faciès typologiques par des méthodes statistiques (analyse factorielle des correspondances et classification ascendante hiérarchique) ne signifie pas nécessairement que ces faciès représentent des entités culturelles ou des phases chronologiques distinctes, ils peuvent également représenter des faciès d'origine fonctionnelle. Nous avons déjà souligné que :

- dans les industries du Gravettien supérieur, à l'exception jusqu'à ce jour de la Picardie et de Plasnn'AI Lomm, la présence des marqueurs gravettiens (pointes de La Gravette et/ou microgravettes) témoigne de l'appartenance de ces techno-complexes à la culture gravettienne ;

- les pointes de La Font-Robert, les éléments tronqués et les burins de Noailles sont associés aux marqueurs gravettiens dans des proportions variables indépendantes de la chronologie ;

- les burins du Raysse se substituant progressivement aux burins de Noailles peuvent également se trouver en association avec les pointes de La Font-Robert et les éléments tronqués (Flageolet, c.VI).

Pour ces raisons, nous avions proposé de ne plus laisser à ces fossiles directeurs la signification chronologique que la séquence de la Ferrassie leur avait fait attribuer (Rigaud 1969, 1982a, 1982b, 1983). À titre d'hypothèse de recherche nous avions alors suggéré (Laville et Rigaud 1973) une interprétation alternative de nature fonctionnelle, en supposant que ces objets avaient été impliqués dans des activités spécifiques à certains sites, certains moments et certains environnements déterminant ainsi leur présence, leur abondance et leur éventuelle association avec d'autres marqueurs. Les variations quantitatives de leur représentation révélant l'intensité, voir l'exclusivité, de ces activités, il n'est pas surprenant de constater qu'il existe des assemblages dans lesquels un de ces objets est largement majoritaire par rapport aux trois autres. C'est notamment le cas :

- des pointes de La Font-Robert à la Ferrassie E1, l'Abri II des Vachons (Sonneville-Bordes 1960);

- des éléments tronqués à La Ferrassie K ( ?) et Grotte XVI c. Abc et Larraux c.5 (ibidem) ;

- des burins de Noailles au Roc de Gavaudun, à l'Abri

Labattut (ibidem), à l'Abri Pataud, c.4 inférieure (David In

(9) II faut remarquer ici que les trois sites « rayssiens » dépourvus de marqueurs gravettiens, La Picardie, Plasenn'Al Lomm et les Artiguaux, sont des sites de plein air et que le site girondin a livré deux burins de Noailles "atypiques ", un grattoir fait sur un fragment de lame à dos abattu, un fragment mésial de lame à dos abattu et un élément tronqué (Lenoir 1997).

(10) Parmi ces moyens, nous avons systématiquement réalisé des diagrammes de projections, procédé aux remontages de l'industrie lithique (Ploux 1986 ; Daulny travaux en cours), suivi les aires de combustions, et pris en compte les résultats de l'étude géologique (J.-P. Texier 1996).

(11) L'absence de niveau gravettien autre que « rayssien » dans la séquence de Solvieux pose la question de l'origine d'une hypothétique pollution du niveau « rayssien » (Sackett 1999 : 299). 


\begin{tabular}{|c|c|c|c|}
\hline & Pataud & & Flageolet 1 \\
\hline GrN 1864 & $18470 \pm 280$ & Ly1606 & $22440 \pm 680$ \\
\hline GrN 1892 & $21540 \pm 160$ & Ly2185 & $18610 \pm 440$ (pollution ?) \\
\hline GrN 4506 & $22780 \pm 140$ & OxA448 & $24600 \pm 700$ \\
\hline GrN 4721 & $23010 \pm 170$ & & \\
\hline OxA 163 & $23180 \pm 670$ & & Le Blot \\
\hline Oxa 164 & $24250 \pm 750$ & Ly 1338 & $24640 \pm 12 \mathrm{O}$ \\
\hline OxA165 & $24440 \pm 740$ & Ly 1339 & $22210 \pm 150$ (pollution? \\
\hline OxA599 & $21740 \pm 450$ & Ly 1340 & $24610 \pm 200$ \\
\hline OxA686 & $24500 \pm 600$ & & \\
\hline
\end{tabular}

Tableau 4 - Datations ${ }^{14} \mathrm{C}$ de quelques niveaux de Gravettien récent.

Table 4 - Radiocarbon dates of some Recent Gravettian assemblages.

Bricker 1995), à l'Abri du Facteur 10-11 (Delporte 1968), au Caillou (Boyer et al. 1984) ;

- les burins du Raysse dans Pataud c. 4 supérieur, La Picardie, Les Artigaux, Plasenn-Al-Lomm. À cet égard, les assemblages très " rayssiens » de ces trois derniers sites de plein air pourraient représenter des faciès dédiés à la mise en oeuvre des «burins » du Raysse.

En revanche, il existe des assemblages où ces fossiles directeurs sont associés en faibles proportions comme au Flageolet c. VII, VI, V, IV (Laville et Rigaud 1973), Roc-deCombe (Bordes et Labrot 1967), les Battuts (Alaux 1973). Cette interprétation fonctionnelle de la variabilité du Gravettien périgourdin qui avait été également soutenue en 1991 par H. Delporte (1991: 251), a reçu récemment une confirmation de la part de B. Aranguren et A. Revedin (2001). Dans une étude du Gravettien supérieur du site de Bilancino (Toscane) qui a livré une industrie à très nombreux burins de Noailles ${ }^{12}$, les résultats conjoints de l'analyse tracéologique et d'une analogie ethnographique ont montré que les burins de Noailles pouvaient avoir été utili- sés dans la préparation de fibres végétales et que leur grand nombre pouvait être mis au compte d'une spécialisation fonctionnelle de cet outillage.

\section{Le Gravettien récent (ex-Périgordien VI)}

II se place au-dessus du Gravettien " noaillien » de l'Abri Pataud et se trouve à la base de la séquence de LaugerieHaute (c'était le «Périgordien III » de D. Peyrony) et, pour cette raison, il est parfois désigné par le terme de « Laugérien ». Le Gravettien récent est caractérisé par la raréfaction des fossiles marqueurs du Gravettien supérieur ${ }^{13}$, un accroissement du nombre des pointes de La Gravette et surtout des microgravettes et par une augmentation sensible du nombre des lamelles à dos (Sonneville-Bordes 1960 ; Bordes 1970 ; Bricker et David 1995 ; Bordes 1968 :69).

Du fait de leur position stratigraphique sur des techno complexes attribués au Gravettien supérieur « noaillien » et « rayssien » surtout, des industries très diverses ont été rapportées à un « Périgordien évolué » équivalent chronologique probable du Gravettien récent : Corbiac 1 (Bordes

(12) Les dates C14-ams suivantes ont été obtenues : $24220 \pm 100$ (Beta 93272), $24970 \pm 110$ (Beta 93271 ) et $25410 \pm 150$ (Beta 106549). Elles montrent clairement la contemporanéité de cette industrie avec les industries à burins de Noailles du sud-ouest de la France.

(13) Quelques rares burins de Noailles sont présents dans des industries contemporaines à Laugerie-Haute, à Pataud, à Corbiac et au Flageolet avec, en outre, dans ce dernier site, un burin du Raysse. 
1967, 1968 ; Bordes et Crabtree 1969), Roc-de-Combe c. 1 (Bordes et Labrot 1967), le Flageolet c. I-III (Rigaud 1982), augmentant ainsi la variabilité de cette industrie qui n'a par ailleurs que peu d'équivalents reconnus. Les datations C14 obtenues pour le Gravettien récent du Sud-Ouest de la France ne confirment que partiellement cette assimilation. De plus, les industries provenant de sites de plein air de Dordogne (Corbiac, Rabier...) n'ayant pu être datées en raison de l'absence totale de matériel organique permettant une datation ${ }^{14} \mathrm{C}$, la position chronologique qui leur fut attribuée n'était fondée de fait que sur des critères technotypologiques qui, à notre avis, ne présentent pas actuellement une fiabilité suffisante pour dépasser le stade d'hypothèse. Pour F. Bordes, "ce Périgordien final ${ }^{14}$ (mais antérieur au Protomagdalénien) est probablement polymorphe : au Roc de Combe comme à Pataud il se trouve sur du Noailles, mais semble bien différent dans ces deux gisements. Il est également possible, il est vrai, que l'un soit antérieur à l'autre. Et tous deux diffèrent de Corbiac, déjà engagé sur la voie protomagdalénienne. » (Bordes 1968).

\section{Le Gravettien final (Protomagdalénien ou Périgordien VII)}

À Pataud comme à Laugerie-Haute, le Gravettien récent est surmonté par le Protomagdalénien qui fut ainsi désigné par $D$. et E. Peyrony (1938) en raison de la composition typologique de l'outillage et de la présence d'un bâton percé en bois de Renne décoré de Mammouths affrontés de facture, selon eux, magdalénienne. L'industrie lithique était largement dominée par les burins, surtout dièdres, et les lamelles à dos qui représentaient près du quart d'un outillage dans lequel quelques rares gravettes et microgravettes attestaient de son caractère gravettien bien qu'il s'en soit écarté par d'autres (SonnevilleBordes 1960 : 224 ; Bordes et Sonneville-Bordes 1966). Dans le Protomagdalénien de l'Abri Pataud, le rapport grattoir/burin est comparable, les lamelles à dos et lamelles à dos tronquées sont également très nombreuses (33 \%) mais les pointes de La Gravette et les microgravettes sont absentes. Outre les lamelles à dos, l'outillage à dos est représenté par de petites lames à dos tronquées et de nombreuses pièces à cran (probables déchets de fabrication de pièces à dos).

Au vu de ces caractères techno-typologiques, Bordes et de Sonneville-Bordes constatant qu'« il n'y a donc, ni dans le style, ni dans les proportions, de rupture nette entre certains Périgordiens supérieurs tardifs et le Protomagdalénien de Laugerie-Haute" (1966 : 121) ont proposé, comme l'avait fait précédemment D. Peyrony (1952), de considérer le Protomagdalénien comme un Périgordien VII et furent sur ce point rejoints par H. Delporte (1991 : 252).

Cependant, cette filiation entre le Gravettien récent (Périgordien VI) et le Protomagdalénien (Périgordien VII) a été l'objet de quelques critiques. D'une part B. Clay (1995: 87) a récusé le terme Protomagdalénien sous le prétexte que l'industrie du niveau 2 de Pataud n'est pas plus « proto» que " magdalénienne » mais sans apporter plus d'arguments et réfute également l'appellation de Périgordien VII dans l'attente de données plus précises. D'autre part, F. Surmely (Surmely, Hays et Costamagno, ce volume) qui repousse une filiation gravettienne et souligne le caractère protomagdalénien de cette industrie au Blot (Virmont 1981 versus Delporte 1991). Si la position de B. Clay est une prudente position d'attente, celle de F. Surmely suscite quelques commentaires. À Laugerie-Haute, les fouilles de D. et E. Peyrony et celles de F. Bordes ont montré, comme celles de H. Delporte au Blot, qu'il y avait quelques liens avec le Gravettien, au moins typologiques, comme en atteste la présence dans le Protomagdalénien de pointes de La Gravette, de microgravettes (et d'un élément tronqué dans la série provenant des fouilles Peyrony). Mettre cette présence de pointes de La Gravette au compte d'un mélange stratigraphique avec les niveaux sous-jacents est une affirmation qui doit être étayée plus sérieusement pour pouvoir servir d'argument. Si la spécificité techno-typologique du Protomagdalénien réside dans la production de grandes lames ${ }^{15}$, une retouche spécifique " forte, mais écailleuse et plus plate que la retouche aurignacienne et l'abondance de microlithes, ces caractères ne sont pas antinomiques avec une filiation gravettienne puisqu'ils sont présents dans le Gravettien récent à Corbiac.

De plus, si en dépit de certains traits gravettiens, cette industrie est un «...faciès culturel [,] distinct du complexe gravettien » et que : « II ne semble donc plus possible de voir dans le Protomagdalénien un "Gravettien final », [...]" (Surmely, ce volume), quelle origine probable pouvonsnous lui trouver? Au vu des caractères techno-typologiques de cette industrie, le Gravettien récent nous semble être la seule réponse acceptable actuellement.

\section{L'origine du Gravettien}

Nous avons présenté plus haut les arguments qui ont établi que le Castelperronien ne pouvait représenter une origine du Gravettien. Pour Otte (1990), les industries gravettiennes les plus anciennes sont celles de Willendorf II, couche 5 où elles sont caractérisées par des micropointes de La Gravette et des fléchettes Des industries semblables proviennent de Geissenklosterle (niveau 1), de Hohle Fels II, de Dolni Vestonice. Les dates ${ }^{14} \mathrm{C}$ pour ces différentes industries sont comprises entre $30500+900 /-800$ et $27000 \mathrm{BP}$. Si l'on accepte l'ancienneté du Gravettien de Willendorf II $(30500+900 \pm 800)$, il est probable que l'Europe a vu le remplacement très rapide des populations aurignaciennes par les groupes gravettiens.

Les travaux de F. Surmely dans le site de Sire à Mirefleurs (Puy-de-Dôme) ont mis au jour une industrie gravettienne ancienne dont l'âge ${ }^{14} \mathrm{C}$ AMS est compris entre $27300 \mathrm{BP}$ et 31305 BP qui situe les occupations de ce site autour de 30000 BP (Surmely, Hays et Costamagno, ce volume) et le Périgordien moyen (Gravettien moyen) de l'Abri Pataud a donné des dates comprises entre 29 et 27000 ans BP. II est

\section{(14) Équivalent du Gravettien récent dans le sens que nous lui donnons ici.}

(15) L'aménagement d'éperon sur le plan de frappe est une technique permettant d'atteindre cet objectif. 
donc probable que le peuplement gravettien de l'Europe centrale et occidentale s'est effectué rapidement, de façon intrusive dans un territoire jusque-là occupé par des populations aurignaciennes dont les témoignages les plus récents sont globalement contemporains des premières manifestations gravettiennes entre 30000 et 29000 ans BP. II convient également de souligner ici que les âges $\mathrm{C} 14$ des dernières manifestations aurignaciennes et des premières occupations gravettiennes se situent dans une période d'approximativement 2000 ans, entre 28000 et $30000 \mathrm{BP}^{16}$. Un tel « recouvrement » qui peut être en partie un artefact technique, pourrait également représenter le temps nécessaire à la disparition des premiers et l'installation des seconds, mais rien n'indique que cela se soit produit de cette façon car, s'il en avait été ainsi, des évidences de contacts auraient certainement été mises au jour. Leur absence plaide donc en faveur d'un remplacement rapide dont témoigne une discontinuité technotypologique brutale et un répertoire symbolique profondément différent. Le Gravettien ancien n'est donc pas connu à ce jour en Aquitaine où la séquence commence par le Gravettien moyen (Périgordien moyen).

\section{Le devenir du Gravettien}

Avec le Protomagdalénien, s'est achevée l'occupation gravettienne. Aussi rapidement qu'elle est apparue dans le nord de l'Aquitaine, la culture gravettienne a été remplacée vers 20000 ans BP (Roques et al. 2001) par le Solutréen qui n'a probablement pas de racines gravettiennes locales ${ }^{17}$. Cependant, D. Peyrony avait fait preuve d'une certaine clairvoyance en proposant le terme de Protomagdalénien en recherchant une référence magdalénienne dans le Magdalénien supérieur plus que dans le Magdalénien ancien (Badegoulien). À titre d'hypothèse pour de futures recherches, nous avions proposé (Rigaud 1976) d'envisager une filiation entre le Gravettien et le Magdalénien " moyen » et " supérieur » (les Magdalénien III à VI de D. de Sonneville-Bordes) en nous fondant sur plusieurs caractères techo-typologiques (pointes de La Gravette et microgravettes, outillage microlithique, technologie des burins) présents sporadiquement dans le Magdalénien et de façon naissante dans le Protomagdalénien. II est évident toutefois, que la filiation entre ces deux cultures sera difficile à établir en raison d'une discontinuité de deux millénaires que semble avoir duré l'occupation solutréenne en Aquitaine septentrionale mais on trouve dans l'Épigravettien du sud-est de la France et du nord de l'Italie, le déve- loppement d'une technologie et d'une typologie qui comble aisément cette lacune.

\section{CONCLUSIONS}

Au cours de ce bilan critique et actualisé sur le Gravettien périgourdin nous avons été conduit à faire quelques remarques d'ordre méthodologique qui jouent à nos yeux un rôle important.

L'archéoséquence régionale, que d'autres appelleront " modèle ", que nous avons proposée se fonde sur des stratigraphies qui, du moins pour les plus longues, ont été revisitées dans la perspective d'une évaluation taphonomique de leur contenu archéologique et paléoenvironnemental ( Delpech, Laville et Rigaud 1984, 1994 ; Texier, Delpech et Rigaud 1999). Ce retour aux sources nous avait été suggéré par de nombreuses discordances dans des données largement utilisées et par des corrélations chronostratigraphiques contestables (Bosselin et Djindjian 1994). En confrontant les données radiométriques, sédimentologiques, palynologiques et paléontologiques disponibles, un certain nombre d'anomalies dans la séquence paléoenvironnementale du Paléolithique supérieur du sud-ouest de la France était devenu évident en particulier dans la zonation pollinique. Nous avions alors recommandé (Delpech, Laville et Rigaud loc. cit.) une prudence extrême dans l'usage, voire même l'abandon, de termes parfois mal définis et imprécis tels que «Kesselt», «Arcy», «Maisières», «Tursac», «Laugerie», «Lascaux» qui peuvent éventuellement correspondre à une réalité locale mais qui ne permettent pas de faire des corrélations à longue distance (Bosselin et Djindjian 1994 versus Rigaud 2000a : 325). On ne peut caractériser une phase climatique, comme l'ont fait ces auteurs (Bosselin et Djindjian 1994 loc. cit :12), sur la base de données polliniques provenant de dépôts dont on sait par ailleurs qu'ils ont subi des perturbations notables dont rendent compte les incohérences des datations ${ }^{14} \mathrm{C}$ (Texier 2001 : 169-172). On peut encore moins corréler cette « tendance climatique » avec une zone pollinique mise en évidence sous des latitudes et dans un milieu différents, pour en déduire finalement une contemporanéité entre deux ensembles archéologiques. C'est la raison pour laquelle nous avons donné une large préférence aux datations radiométriques et plus particulièrement aux datations ${ }^{14} \mathrm{C} A M S^{18}$ et à la biostratigraphie dans l'établissement du cadre chronologique des industries gravettiennes périgourdines (voir Delpech et Texier, ce volume).

(16) La date de l'Aurignacien évolué du niveau 6-lentille 1 de Pataud $24340 \pm 700$ (OxA 582) est à l'évidence suspecte. Les dates obtenues pour l'Aurignacien du niveau 7-lentille W-1 semble plus conforme à la réalité ; elles sont comprises entre $29300 \pm 450$ (GrN3105) et $32900 \pm 700$ (GrN 3116). Au Flageolet, l'Aurignacien le plus récent a donné des âges de $24800 \pm 600$ (OxA 597), $26800 \pm 1000$ (Ly 2724) et $28970 \pm 750$ (GifA 95 540). Le niveau Aurignacien de la Grotte XVI a été daté de $28140 \pm 405$ (AA 6840), $29285 \pm 420$ (AA6841) et $29740 \pm 510$ (GifA 94201).

(17) La présence d'un niveau d'« Aurignacien $V$ » pose le problème de son existence réelle et de son attribution à l'Aurignacien (Aubry et al. 1995).

(18) Les datations ${ }^{14} \mathrm{C}$ AMS étant faites sur un échantillon ponctuel et précisément localisé dans l'espace constituent des données largement préférables aux datations faites anciennement sur les échantillons constitués de fragments d'os ou de bois provenant de l'ensemble d'un niveau. 


\begin{tabular}{|c|c|c|c|c|c|}
\hline D. Peyrony & $\begin{array}{l}\text { F. Bordes et D. de } \\
\text { Sonneville-Bordes }\end{array}$ & H. L. Movius et al. & H. Delporte & $\begin{array}{l}\text { F. Djindjian et B. } \\
\text { Bosselin }\end{array}$ & J.-Ph. Rigaud \\
\hline \multirow[t]{2}{*}{$\begin{array}{l}\text { Protomagdalénien ou Périgordien } \\
\text { VII }\end{array}$} & $\begin{array}{l}\text { Protomagdalénien ou } \\
\text { Périgordien VII }\end{array}$ & Protomagdalénien & Protomagdalénien & Protomagdalénien & $\begin{array}{l}\text { Protomagda - } \\
\text { lénien } \\
\text { ou Gravettien } \\
\text { final } \\
\end{array}$ \\
\hline & $\begin{array}{l}\text { Périgordien VI } \\
\text { ex-Périgordien III }\end{array}$ & Périgordien VI & Périgordien supérieur & Laugérien & $\begin{array}{l}\text { Gravettien } \\
\text { récent }\end{array}$ \\
\hline Périgordien Vc & Périgordien Vc & \multirow[t]{3}{*}{$\begin{array}{l}\text { Noaillien ou } \\
\text { Périgordien Vc }\end{array}$} & $\begin{array}{l}\text { Périgordien supérieur à } \\
\text { burins de Noailles }\end{array}$ & $\begin{array}{l}\text { Rayssien } \\
\text { Noaillien }\end{array}$ & \multirow{3}{*}{ 总 } \\
\hline Périgordien $\mathrm{Vb}$ & Périgordien $\mathrm{Vb}$ & & $\begin{array}{l}\text { Périgordien supérieur à } \\
\text { éléments tronqués }\end{array}$ & $\begin{array}{l}\text { Gravettien } \\
\text { indifférencié }\end{array}$ & \\
\hline Périgordien Va & Périgordien Va & & $\begin{array}{c}\text { Périgordien supérieur à } \\
\text { pointes de la Font Robert } \\
\text { ou } \\
\text { Fontirobertien }\end{array}$ & \multirow[t]{2}{*}{ Fontirobertien } & \\
\hline Périgordien IV & Périgordien IV & Périgordien moyen & Bayacien & & $\begin{array}{c}\text { Gravettien } \\
\text { moyen }\end{array}$ \\
\hline \multicolumn{6}{|l|}{ Périgordien III } \\
\hline Périgordien II & Aurignacien? & & & & \\
\hline $\begin{array}{l}\text { Périgordien I } \\
\text { ou ancien }\end{array}$ & Périgordien ancien & Périgordien inférieur & & & \\
\hline
\end{tabular}

Tableau 5 - Tableau montrant les correspondances entre les modèles successifs proposés pour le Gravettien du nord de I 'Aquitaine.

Table 5 - Correspondences between the successive proposed models for the Gravettian in northern Aquitaine.

En outre, il est évident que l'étude des cultures préhistoriques doit se faire dans le contexte le plus large et pour le Gravettien l'échelle européenne s'impose. Toutefois, avant de s'engager dans des synthèses qui risquent de paraître prématurées et hasardeuses, il est indispensable de s'appuyer sur un corpus de séquences paléoenvironnementales et archéologiques régionales rigoureuses (cf. supra).

Par leur composition typologique, certaines industries ont montré des caractères originaux qui se sont exprimés par des appellations distinctes (Bayacien, Fontirobertien, Noaillien, Rayssien, Laugerien, etc) qui n'ont pas, selon les auteurs, la même signification. Ces entités taxinomiques n'ont pas de réelle signification culturelle. Elles représentent des faciès techno-typologiques auxquels nous avons donné une probable origine fonctionnelle.
En bref, l'occupation gravettienne du nord de l'Aquitaine s'est réalisée à partir de 29000 ans. Elle fut relativement rapide et l'absence de réelles industries mixtes ${ }^{19}$, présentant à la fois des caractères aurignaciens et gravettiens, semble indiquer qu'il n'y a eu ni transformation locale de l'aurignacien, ni contemporanéité locale de ces cultures. Le Gravettien est arrivé en Aquitaine avec des spécificités techniques, typologiques et symboliques accomplies, acquises et développées préalablement à son arrivée sur la façade atlantique de l'Europe pendant le Gravettien ancien. Les premières manifestations gravettiennes en Aquitaine sont donc attribuables à un Gravettien moyen.

Par la suite, très rapidement, au cours d'une période de l'ordre d'un millénaire, le répertoire typologique et technologique du Gravettien s'est enrichi et diversifié dans le contexte climatique instable préalable à la mise en place

(19) Et non des mélanges stratigraphiques. 
du dernier maximum glaciaire. Le Gravettien supérieur, largement représenté dans le sud de la France et l'ouest de I'Italie, semble n'avoir fait que de faibles et brèves incursions dans les zones plus septentrionales.

Le Gravettien récent se caractérise par l'abandon progressif des marqueurs du stade précédent et par un retour à des équipements moins diversifiés plus proches du Gravettien moyen.

Cette tendance se confirmera par la suite au cours du Gravettien final dont le caractère transitionnel vers des techno-complexes dont les affinités avec le Magdalénien moyen et supérieur sont indéniables.

Nous avons mis en correspondance dans le tableau 5, les modèles interprétatifs successifs de la séquence gravettienne dans le nord de l'Aquitaine. Cette archéoséquence est une contribution régionale à l'établissement d'un modèle européen qui se doit d'intégrer l'ensemble des données régionales.

\section{BIBLIOGRAPHIE}

ALAUX J.-F. 1973 - Pointes de La Font-Robert en place dans le Périgordien à burins de Noailles de l'abri des battuts (commune de penne (Tarn). Bull. Soc. Préhist. Fr. 51 :55.

AUBRY T., DETRAIN L. et KERVAZO B. 1995 - Les niveaux intermédiaires entre le Gravettien et le Solutréen de l'abri Casserole (Les Eyzies-de-Tayac) : Mise en évidence d'un mode de production originale de microlithes et implications. Bull. Soc. Préhist. Fr., 92, n 3 : 296-301.

ARANGUREN B. et REVEDIN A. 2001 - Interprétation fonctionnelle d'un site gravettien à burins de Noailles. L'Anthropologie, 2001, vol. 105, n 4 : 533-545.

BAZILE F. 2005 - Un rare campement Gravettien. Archeologia, 419 : 59-67.

BORDES F. 1967.- Considérations sur la typologie et les techniques dans le Paléolithique. Quartär, bd 18, 1967 : 25-57, $8 \mathrm{PI}$.

BORDES F. 1968 - La question périgordienne. In : La Préhistoire problèmes et tendances, CNRS éd. Paris, 1968, p. 59-70.

BORDES F. 1970 - Observations typologiques et techniques sur le Périgordien supérieur de Corbiac (Dordogne). Bull. Soc. Préhist. Fr., t. 67 : 105-113.

BORDES F. and CRABTREE D. 1969 - The Corbiac blade technique and other experiments. Tebiwa, vol. 12, $\mathrm{n}^{\circ} 2$, $1969: 1-21$.

BORDES F. et LABROT J. 1967 - La stratigraphie du gisement de Roc-de-Combe (Lot) et ses implications. Bull. Soc. Préhist. Fr. 64 : 15-28
BOSSELIN B. et DJINDJIAN F.1994 - La chronologie du Gravettien français. Préhistoire européenne, Liège, 1994, vol.6, 77-115.

BOYER M., GENESTE J.-M. et RIGAUD J.-Ph. 1984 - Le Périgordien supérieur du site de plein air du Caillou, Rouffignac-de-Sigoulès (Dordogne). Bull. Soc. Préhist. Fr. 1984, p. 302-310.

BRICKER H. 1978 - Lower to Middle Perigordian continuity. In : Giardino M.J. et al eds. Human Mosaïc, $\mathrm{n}^{\circ}$ 12. New Orleans, $1978:$ 165-182.

BRICKER H. M. (Dir.) 1995 - Le Paléolithique supérieur de l'Abri Pataud (Dordogne) : les fouilles de H. L. Movius Jr. Suivi d'un inventaire analytique des sites aurignaciens et périgordiens de Dordogne. DAF 50, Paris : MSH, 1995.

BRICKER H. 1995a - Le Périgordien moyen de l'Abri Pataud niveau 5. In : Bricker H. M. (Dir.), 1995 Le Paléolithique supérieur de I 'Abri Pataud (Dordogne) : les fouilles de H. L. Movius Jr. Suivi d'un inventaire analytique des sites aurignaciens et périgordiens de Dordogne. DAF 50, Paris : MSH, 1995.

BRICKER H. et DAVID N. 1995 - Le Périgordien VI de l'Abri Pataud, niveau 3. In : Le Paléolithique supérieur de l'Abri Pataud (Dordogne). Les fouilles de H.L. Movius Jr., Paris : MSH, 1995, DAF $50:$ 89-104.

CÉLÉRIER G. 1967 - Le gisement périgordien supérieur des « Jambes ", commune de Périgueux (Dordogne). Bull. Soc. Préhist. Fr. 64 : 53-68.

CLAY R.B. 1995 - Le Protomagdalénien de l'Abri Pataud, niveau 2. In : Le Paléolithique supérieur de l'Abri Pataud (Dordogne). Les fouilles de H.L. Movius Jr., Paris : MSH. 1995, DAF 50 : 67-88.

DAVID N. 1985 - Excavation of the Abri Pataud,Les Eyzies (Dordogne) : the Noaillian (level 4) assemblage and the Noaillian culture in Western Europe. Cambridge : Harvard University Peabody Museum, 1985.

DAVID N. 1995 - Le Noaillien (Périgordien Vc) de l'Abri Pataud, niveau 4, éboulis 3-4 : Moyen + inférieur, niveau 4a, in : Le Paléolithique supérieur de l'Abri Pataud (Dordogne). Les fouilles de H.L. Movius Jr., Paris : MSH 1995, DAF $50: 105-132$.

DELIBRIAS G. 1984 - La datation par le carbone 14 des ossements de La Ferrassie, in : Delporte H. : Le grand abri de La Ferrassie. Fouilles 1968-1973. Etudes Quaternaires 7. Publication de I'Université de Provence : 105-107.

DELPECH F. et RIGAUD J.-Ph. 2001 - Quelques exemples de l'apport des datations en archéologie préhistorique. In : Barandon J.-N., Guibert P. et Michel V. dir.. Datation, APDCA Ed. Antibes 2001 : 315-331. 
DELPECH F., LAVILLE H., RIGAUD J.-Ph. 1984 - Sur la zonation pollinique du Pléistocène récent : les précisions du domaine aquitain. Journées de Palynologie archéologique, 25-26-27 janvier 1984, Valbonne.

DELPECH F., LAVILLE H., RIGAUD J.-Ph. 1994 Chronologie et environnement climatique du Paléolithique supérieur dans le sud-ouest de la France. In : El cuadro geocronologico des Paleolitico superior initial (F.B. de Quiros, dir.), Museo y centro de investigacion de Altamira, Monografia $n^{\circ} 13: 173-186$.

DELPORTE H. 1955 - L'industrie de Châtelperron et son extension géographique. Congrés Préhistorique de France, 14, Strasbourg-Metz (1953), 233-249.

DELPORTE H. 1968 - L'abri du facteur à Tursac. Gallia Préhistoire, $11: 1-112$.

DELPORTE H. 1984 (Dr) - Le Grand Abri de La Ferrassie. Fouilles 1968-1973. Etudes Quaternaire 7 ; Publication de l'Université de Provence.

DELPORTE H. 1991 - La séquence aurignacienne et gravettienne sur la base de travaux récents réalisés en Périgord. Bull. Soc. Préhist. Fr., t. 88 (8) : 243-256.

DELPORTE H. et TUFFREAU A. 1972-1973 - Les industries du Périgordien supérieur de La Ferrassie. Quartär 23/24 : 93-123.

DIBBLE H. L. and LENOIR M. 1995 - The Middle Palaeolithic site of Combe-Capelle Bas (France). The University Museum monograph 91, University of Pennsyllvania, 1995.

DJINDJIAN F. et BOSSELIN B. 1994 - Périgordien et Gravettien : l'épilogue d'une contradiction ? Préhistoire européenne, Liège, 1994, vol.6 : 117-131.

DJINDJIAN F., KOZLOWSKI J et OTTE M. 1999 - Le Paléolithique supérieur en Europe. Paris, Armand Colin, 1999.

GAROD D. 1938 - The Upper Palaeolithic in the light of recent discovery. Proceding of the Prehistoric Society 4 : 1-26.

GOUTAS N. 2004 - Caractérisation et évolution du Gravettien en France par l'approche techno-économique des industries en matières dures animales (étude de six gisements du Sud-ouest). Thèse de doctorat de Préhistoire, Université Paris 1.

GUILLERMIN P. (ce volume) - Les «Périgordiens » en Quercy : l'exemple du gisement des Fieux.

HAESERTS P. ET HENZELIN J. de 1979 - Le site paléolithique de Mazières-Canal. Dissertationes Archaeologicae Gandenses, XIX, 120 p, 44 fig., 20 pl.

HARROLD F. B. 1989 - Mousterian, Chatelperronian and Aurignacian in western Europe : continuity or discontinuity.
In : The Human revolution : behavioural and biological perspectives on the origines of modern humans (eds P. Mellars \& C. Stringer), Edingburg University Press, p. 677-713.

KLARIC J. 2003 - L'unité technique des indistries à burins du Raysse dans leur contexte diachronique. Réflexion sur le diversité culturelle au Gravettien à partir des données de la Picardie, d'Arcy-sur-Cure, de Brassempouy et du Cirque de la Patrie. Thèse de Doctorat de Préhistoire, Université de Paris 1.

LACORRE F. 1960 - La Gravette, le Gravettien et le Bayacien. Laval : imprimerie Barnéou, 1960.

LAVILLE H. et RIGAUD J.-Ph. 1973 - The Perigordian V industries in Perigord : typological variations, stratigraphy and relative chronology. World Archaeology, vol. $4, n^{\circ} 3$ : 330-338.

LENOIR M. 1997 - Un gisement de plein air du Périgordien supérieur en Gironde : Les Artigaux à Camiac et Saint-Denis. Bull. Soc. Préhist. Fr. t 74, 1977, Etudes et Travaux, fasc. 2.

LUCAS G. 2000 - Les industries lithiques du Flageolet1 (Dordogne) : approche économique, technologique, fonctionnelle et analyse spatiale (Aurignacien et Périgodien). Thèse Université Bordeaux 1.

MELLARS P. 1989 - Major issues in the emergence of modern human. Current anthropology, 23, 238-40.

MOVIUS H. L. Jr. 1954 - Les Eyzies, A test Excavation. Archaeology, 7, 1954, 82-90.

MOVIUS H. L. 1977 - Excavations at the Abri Pataud, Les Eyzies, Dordogne : Stratigraphy. American school of prehistoric research. Peabody museum , Harvard University, Bull. $\mathrm{n}^{\circ} 13$.

O'FARELL M. 1996 - Approche technologique et fonctionnelle des pointes de La Gravette, une analyse archéologique et expérimentale appliquée à la collection de Corbiac (Dordogne). Mémoire de DEA, Université Bordeaux 1.

OTTE M. 1979 - Le Paléolithique supérieur ancien en Belgique. Bruxelles, 684 p., 256 fig.

OTTE M. 1990 - Révisions de la séquence du Paléolithique supérieur de Willendorf (Autriche). Bulletin de l'Institut Royal des Sciences Naturelles de Belgique, Bruxelles, 1990, t. $60: 219-228$.

OTTE M. et NOIRET P. 2003 - L'Europe Gravettienne in Préhistoire de l'Europe, des origines à l'âge du bronze. $\mathrm{R}$. Desbrosse et A. Thévenin (éd.). CTHS, Paris 2003.

PEAN S. 2001 - Comportements de subsistance au Gravettien en Europe centrale (Autriche, République Tchèque, Pologne, Hongrie). Thèse de doctorat du Museum national d'Histoire Naturelle (01 MNHN 0008), M. Patou-Mathis, dir. 
PEYRONY D. 1933 - Les industries aurignaciennes dans le bassin de la Vézère, Aurignacien et Périgordien. Bull. Soc. Préhist. Fr., t. 30 (10),1933 : 543-559.

PEYRONY D. 1934 - La Ferrassie. Préhistoire, t. III.

PEYRONY D. 1943 - Le gisement du Roc de Combe Capelle (Commune de Saint Avit-Sénieur) Dordogne. Bulletin de la Société Historique et Archéologique du Périgord, t. LXX : 158-175.

PEYRONY D. 1952 - Correspondance. Bull. Soc. Préhist. Fr., t. 49, p. 567.

PEYRONY D. et E. 1938 - Laugerie-Haute. Archives de I'Institut de Paléontologie Humaine, Mémoire $\mathrm{n}^{\circ} 19$.

PLOUX S. 1986 - Essai d'interprétation d'une concentration lithique : technologie et remontage, couche $\mathrm{Vl} d u$ Flageolet 1 à Bézenac (Dordogne). Gallia Préhistoire, t 29, $\mathrm{n}^{\circ} 1$, p. 29-62.

RIGAUD J.-Ph. 1969 - Note sur la stratigraphie du gisement du Flageolet 1, commune de Bézenac, Dordogne. Bull. Soc. Préhist. Fr., t. 66 : 73-75.

RIGAUD J.-Ph. 1976 - Les civilisations du Paléolithique supérieur en Périgord. La Préhistoire Française, t. 1.2. Paris, C.N.R.S., 1976 : 1257-1270.

RIGAUD J.-Ph. 1976 - Données nouvelles sur le Périgordien en Périgord. IXè congrés de l'UISPP, colloque XV : Périgordien et Gravettien en Europe, Nice, 13-18 septembre 1976.

RIGAUD J.-Ph. 1978 - The significance of variability among lithic artefacts : a specific case from Southwestern France. Journal of Anthropological Research, fall 1978, vol. $34, n^{\circ} 3: 299-310$.

RIGAUD J.-Ph. 1982a - Données nouvelles sur l'Aurignacien et le Périgordien en Périgord. ERAUL, Liège, t. $13-2: 289-324$.

RIGAUD J.-Ph. 1982b - Le Paléolithique supérieur en Périgord : les données du Sud-Ouest sarladais et leurs implications. Bordeaux, Université Bordeaux 1, 1982, 2 t., Thèse de Doctorat d'Etat ès Sciences.

RIGAUD J.-Ph. 1983 - Données nouvelles sur le Périgordien supérieur en Périgord. ERAUL, Liège, t. 13-1 : 107-118.

RIGAUD J.-Ph. 1985 - Réflexions sur la signification de la variabilité des industries lithiques paléolithiques. In : OTTE, M. (Ed.) La signification culturelle des industries lithiques : actes du colloque de Liège, 3-7 octobre 1984. Oxford : B.A.R., 1985, p. 374-390. Studia Praehistorica Belgica ; 4 : B.A.R. International series ; 239.

RIGAUD J.-Ph. 1988 - The gravettian peopling of southwestern France : Taxonomic problems. In : Upper Pleistocene Prehistory of Western Eurasia. University
Museum Symposium Series 1, Monograph 54. The University Museum, University of Pennsylvania.

RIGAUD J.-Ph. 1994 - L'évaluation contextuelle préalable à l'analyse de la répartition spatiale des vestiges. Préhistoire et Anthropologie Méditerranéennes, 1994, T. 3, p. 39-41.

RIGAUD J.-Ph. 2000a - Human adaptation to the climatic deterioration of the Last Pléniglacial in the Southwestern France (30 $000-20000$ BP) in Hunters of the Golden Age. The Mid-Upper Paleolithic of Eurasia $30000-20000$ BP. W. Roebroeks, M. Mussi, J. Svoboda et K. Fennma eds University of Leiden,2000.

RIGAUD J.-Ph. 2000b - Late neanderthals in the south west of France and the emeergence of the Uppel Palaeolithic in Neanderthals on the edge (Stringer, Barton \& Finlayson eds.) Oxbow Books, Oxford, 2000. Conference marking the $150^{\text {th }}$ anniversaire of the Forbe' Quarry discovery, Gibraltar, Spring 1998 : 27-31.

RIGAUD J.-Ph. et SIMEK J. 1993 - La grotte XVI. In : Bilan scientifique de la région aquitaine, 1992. Min. Culture et Patrimoine, Sous-direction de l'Archéologie, DRAC Aquitaine, p. 24.

ROQUE C., GUIBERT P., VARTAGNAN E., BECHTEL F., OBERLIN C., EVIN J., MERCIER N., VALLADAS H., TEXIER J.-P., RIGAUD J.-PH., DELPECH F., CLEYETMERLE J.-J. et TURQ A. 2001 - Une expérience de croisement de datations $\mathrm{TL}^{14} \mathrm{C}$ pour la séquence solutréenne de Laugerie-Haute, Dordogne. In : J.N. Barrandon, P. Guibert, V. Michel (sous la dir. de), Datation. XXlè Rencontres Internationales d'Archéologie et d'Histoire d'Antibes. - Antibes : Editions APDCA, 2001, pp. 217-232.

SACKETT J. 1999 - The Archaeology of Solvieux. An Upper Palaeolithic Open Air site in France. Monumenta archaeologica 19. Institute of Archaelogy, University of California, Los Angeles.

SONNEVILLE-BORDES D. (de) 1955a - La question du Périgordien II. Bull. Soc. Préhist. Fr. 1955 :187-203.

SONNEVILLE-BORDES D. (de) 1955b - A propos du Périgordien. Bull. Soc. Préhist. Fr. 1955 :597-601.

SONNEVILLE-BORDES D. (de) 1955c - A propos du Périgordien II. Bull. Soc. Préhist. Fr. 1955 :663-665.

SONNEVILLE-BORDES D. (de) 1960 - Le Paléolithique supérieur en Périgord. Delmas, Bordeaux 1960.

SONNEVILLE-BORDES D. (de) 1966 - L'Evolution du Paléolithique supérieur en Europe occidentale et sa signification. Bull. Soc. Prehist. Fr., t. 63, Etudes et Travaux, $\mathrm{n}^{\circ} 1: 3-34$.

SORIANO S. 1998 - Les microgravettes du Périgordien de Rabier à Lanquais (Dordogne). Analyse technologique fonctionnelle. Gallia Préhistoire, 40, 1998, 75-94. 
SURMELY F., HAYS M. COSTAMAGNO S. (ce volume) - Le Gravettien et le Protomagdalénien en Auvergne.

TEXIER J.-P. 1996 - The deposits of Le Flageolet (Dordogne, France) : dynamic protestes, stratigraphie and Paleoenvironnemental interprétation. In : Simek and Rigaud, Symposium :The Early upper Palaeolithic of Le Flageolet 1. 61st annual meeting of the Society for American archaeology, 10-14 April 1996, New Orleans.

TEXIER J.-Ph. 2001 - Sédimentogénèse des sites préhistoriques et représentativité des datations numériques. In : Barandon J.-N., Guilbert P. et Michel V. dir. Datation, APDCA Ed. Antibes 2001: 159-175.
TEXIER J.-P., DELPECH F. et RIGAUD J.-Ph. 1999 Programme collectif de recherche " litho- et bio-stratigraphique de quelques sites paléolithiques de référence du Périgord », Rapport final,SRA Aquitaine, 38 p.

VIRMONT J. 1981 - Le bassin de l'Allier au Paléolithique supérieur. Industries et phases de peuplement. Thèse de $3^{\text {ème }}$ cycle de l'Université d'Aix-Marseille, $378 \mathrm{p}$. 
